\title{
Inhibitors of quinolinic acid synthesis: new weapons in the study of neuroinflammatory diseases
}

\begin{abstract}
"The representation of QUIN as a mere product of the Trp catabolism on route towards the essential cofactors nicotinic acid and NAD, largely underestimates, however, the variety of the physiological role played by , pathophysiological processes.
\end{abstract}

Keywords $\bullet$ inhibitors $\bullet$ kynurenine pathway $\bullet$ neuroinflammation $\bullet$ neuroprotection - quinolinic acid

There is no doubt that quinolinic acid (QUIN) is an interesting molecule. QUIN is endowed with a kaleidoscopic landscape of properties and physiological implications, and offers a continuously increasing number of potential therapeutic applications for modulators of its synthesis and metabolism.

The aim of this article is not to review the many physiological implications of QUIN [1-3], but rather to offer some alternative (and even provocative) views that can stimulate interest from different perspectives and to suggest still unexplored ways to understand the role of QUIN in the CNS as well as in peripheral diseases.

QUIN is synthesized through the socalled kynurenine pathway (KP) of tryptophan metabolism, and as such is a product of the oxidative metabolism of tryptophan (Trp). The representation of QUIN as a mere product of the Trp catabolism on route towards the essential cofactors nicotinic acid and NAD, largely underestimates, however, the variety of the physiological role played by QUIN and its involvement in pathophysiological processes.

\section{QUIN as a neurotoxin}

It was more than three decades ago that QUIN was discovered to be a neuroactive compound. The activity of QUIN in the CNS certainly deserves mention, also in view of the growing therapeutic opportunities endowed with the modulation of its synthesis and metabolism.
After the first observation by Lapin et al. that an intracerebreventricular injection of QUIN produced convulsions, Stone et al. first characterized it as an NMDA agonist in 1981 [4]. The neurotoxic properties of QUIN, and perhaps its role as a true endogenous excitotoxin were further elaborated in the mid1980s, following the seminal work of Schwarcz et al., who described the peculiar features of QUIN as a plausible excitotoxic agent [5,6]. Thus, it was clearly demonstrated that QUIN produces a pattern of lesions that are specific for striatum, and which closely resemble those observed in the brain of patients with Huntington's disease [7]. Interestingly, this was put in relation to the intriguing observation (rather obvious in many respects, but never fully exploited from a medicinal chemistry point of view) that despite being characterized pharmacologically as an excitatory amino acid endogenous agonist, QUIN in fact is not an amino acid [8].

Understanding the role of QUIN in the brain demanded clarification of the role, function and significance of its synthesizing pathway, namely the KP. The KP is now characterized as the center of an intricate signaling network linking together immune, adaptive and neurological functions. In particular, besides QUIN at least two other metabolites along the pathway have pronounced CNS activity, namely kynurenic acid (KYNA), a noncompetitive NMDA antagonist and $\alpha 7$ nicotinic receptor antagonist, and 3-hydroxy-kynurenine (3-HK), a

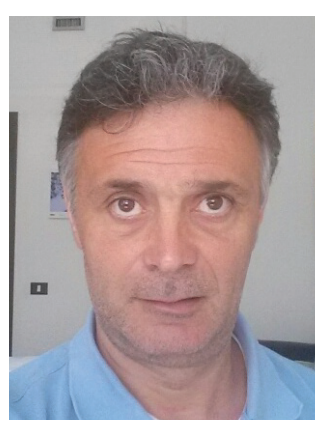

Gabriele Costantino* *Dipartimento di Farmacia- Viale Area delle Scienze, 27/A. Università degli Studi di Parma, 43124 Parma, Italy Tel.: +390521905055

gabriele.costantino@unipr.it 
pro-excitotoxic and pro-oxidant metabolite. KYNA and QUIN are produced along two different branches of the KP, and these two branches are also physically segregated in the brain [9], with the QUIN branch being expressed in microglia, whereas the KYNA arm is present in astrocytes, reflecting the low abundance of KMO, the enzyme producing 3-HK and then QUIN in this cellular type. The presence of a 'good' and 'bad' arm of the KP has constituted the conceptual basis upon which the search for KP modulators as neuroprotective agents has been based over the past 20 years. Thus, having recognized the QUIN branch of the KP as the neurotoxic component, and the KYNA arm the neuroprotective one, most approaches pursued over the last two decades to achieve neuroprotection were based on attempts to unbalance the pathway towards KYNA, by inhibiting the first enzyme of the QUIN pathway, namely $\mathrm{KMO}[10,11]$. This editorial further aims to critically analyze this approach, and speculate as to whether targeting enzymes directly synthesizing (or metabolizing) QUIN can be a more productive one.

\section{"The presence of a 'good' and 'bad' arm of the KP has constituted the conceptual basis upon which the search for KP modulators as neuroprotective agents has been based over the past 20 years.}

\section{QUIN is at the center of an intricate network of players in the immune response}

It is interesting to note that despite the amount of research carried out over the past 20 years to understand the physiopathological role of the KP, and in spite of the observation that most of the enzymes of pathway have been characterized in detail from a molecular point of view, including the availability of high-resolution crystal structures for many of them, the biological significance of individual enzymes is still poorly understood. This is, in part, due to the lack of suitable chemical probes to be used to check the relevance of individual steps of the KP in a cellular or in vivo context. In a vicious cycle this has, in turn, hampered the exploitation of individual enzymes in therapeutically useful ways. This is particularly true for enzymes directly involved in the synthesis or in the metabolism of QUIN, which have been scarcely characterized pharmacologically due to a lack of suitable inhibitors. Let us focus, for example, on 3-hydroxyanthranilate dioxygenase (3-HAO), the enzyme that converts 3-hydroxyanthranilic acid into QUIN. So far, most of the approaches aimed at reducing QUIN production were based on KMO inhibitors, on the assumption that $\mathrm{KMO}$ inhibition would result in KYN accumulation thus shifting the pathway towards the neuroprotective KYNA arm. This approach has never been translated into investigational drugs for many reasons that are too long to discuss here, but what should be stressed is that a QUIN-centric view of the pathway may also offer an alternative perspective of the possible therapeutic applications. The direct precursor of QUIN is 3-hydroxyanthranilic acid (3HAA). It is quite apparent that inhibition of $\mathrm{KMO}$, or inhibition of 3-HAO has an opposite result in the biosynthesis of this intermediate. While both approaches should result in a decreased QUIN synthesis, the former also decreases 3HAA, while the latter would increase it. The difference may not seem so important if one focuses on the KYN-KYNA-QUIN paradigm, but it becomes fundamental if one starts appreciating the role of 3 -anthranilic acid (AA) and of its metabolites in neuroimmunological responses. In this context, it is now recognized that 3-AA plays an important role in the suppression of T-cell response [12], and this mechanism has been linked to the development of the immunetolerance after IDO activation. These may have a very different application in diseases where activation of the immune system has the opposite result, for example, in cancer or in autoimmune diseases. Even more interesting is the fact that 3HAA is converted nonenzymatically in cinnabarinic acid (CINNA), a dimeric product of 3HAA oxidation. Considered as a metabolic curiosity, CINNA is now recognized as an important modulator of the immune system and as a potential neuroprotective agent, in view of its ability to activate the mGluR 4 subtype of the metabotropic glutamate receptors [13]. Thus, blocking the QUIN branch upstream of the KP would decrease not only the neurotoxic QUIN, but also the neuroprotective CINNA and the immune competent 3HAA. Finally, it is important to mention two neglected players in the KP, namely kynureninase and quinolonic acid phosphoribosyltransferase (QPRT). The first hydrolyses KYN into AA (but also 3-HK into 3HAA) and the potential for its selective inhibition should be carefully considered in the light of the observation that the ratio between AA and 3AA is an important, though still obscure, marker in several neuroinflammatory diseases. The second, QRPT, is the enzyme that starts the second group of reactions leading to NAD+ from QUIN. Since NAD + is an essential cofactor for cell viability, inhibiting the QUIN branch of the KP under neuroinflammatory conditions may seem a counterintuitive approach, since the resulting decrease in NAD+ should confer reduced resistance to oxidative stress. However, QPRT becomes saturated at relatively low concentrations (300-500 nM), while QUIN displays neurotoxicity above $500 \mathrm{nM}$. Thus, 
the 'ideal' QUIN synthesis inhibitor should be able not to completely block the QUIN synthesis but rather to equilibrate its concentration just below the physiological one. It should also be observed that QPRT may exert a protective role in malignant cells. For example, gliomas may show increased resistance to oxidative stress or chemo- or radio-therapy because they can use QUIN and QPRT as an alternative source of NAD+. Inhibiting QPRT may prove to be a promising strategy to better exploit chemo- or radio-treatment of gliomas by increasing their susceptibility to treatment [14].

\section{A strong need for chemical tools}

The QUIN-centric analysis of the KP of the Trp metabolism proposed above suggests that the potential for KP modulation is not limited to enzymes classically considered for neuroprotection, but can be further expanded by better understanding the pathophysiological role of 'neglected' enzymes. It is surpris-

\section{References}

1 Stone TW, Stoy N, Darlington LG. An expanding range of targets for kynurenine metabolites of tryptophan. Trends Pharmacol. Sci. 34(2), 136-143 (2013).

2 Schwarcz R, Bruno JP, Muchowski PJ, Wu HQ. Kynurenines in the mammalian brain: when physiology meets pathology. Nat. Rev. Neurosci. 13(7), 465-477 (2012).

3 Guillemin GJ. Quinolinic acid, the inescapable neurotoxin. FEBS J. 279(8), 1356-1365 (2012).

4 Stone TW, Perkins MN. Quinolinic acid: a potent endogenous excitant at amino acid receptors in CNS. Eur. J. Pharmacol. 72, 411-412 (1981)

5 Schwarcz R, Köhler C. Differential vulnerability of central neurons of the rat to quinolinic acid. Neurosci. Lett. 38, 85-90 (1983).

6 Whetsell WO Jr, Schwarcz R. Prolonged exposure to submicromolar concentrations of quinolinic acid causes excitotoxic damage in organotypic cultures of rat corticostriatal system. Neurosci. Lett. 97, 271-275 (1989).

7 Beal M, Kowall N, Ellison D et al. Replication of the neurochemical characteristics of Huntington's disease by quinolinic acid. Nature 321, 168-171 (1986).

8 Schwarcz R, Guidetti P, Sathyasaikumar KV, Muchowski PJ. Of mice, rats and men: revisiting the quinolinic acid hypothesis of Huntington's disease. Prog. Neurobiol. 90 (2), 230-245 (2010).

9 Amori L, Guidetti P, Pellicciari R, Kajii Y, Schwarcz R. On the relationship between the two branches of the kynurenine pathway in the rat brain in vivo. J. Neurochem. 109(2), 316-325 (2009). ing that the repertoire of chemical tools to be used as probes to understand their function is so limited for enzymes otherwise well characterized in terms of structure and in cellular localization. Only recently a class of 3-HAO inhibitors, chemically stable and active in vivo, has been reported [15]. The challenges that the enzymes such as 3-HAO, kynureninase or QPRT offer to medicinal chemists are significant, as are the promises in their selective inhibition.

\section{Financial \& competing interests disclosure}

This work was supported by grants PRIN2010-11 from the Ministero dell' Istruzione, Università e Ricerca (Italy). The author has no other relevant affiliations or financial involvement with any organization or entity with a financial interest in or financial conflict with the subject matter or materials discussed in the manuscript apart from those disclosed.

No writing assistance was utilized in the production of this manuscript.

Pellicciari R, Natalini B, Costantino G et al.Modulation of the kynurenine pathway in search for new neuroprotective agents. Synthesis and preliminary evaluation of (m-nitrobenzoyl)alanine, a potent inhibitor of kynurenine-3hydroxylase. J. Med. Chem. 37(5), 647-655 (1994).

11 Röver S, Cesura AM, Huguenin P, Kettler R, Szente A. Synthesis and biochemical evaluation of $\mathrm{N}$-(4-phenylthiazol$2-y l$ ) benzenesulfonamides as high-affinity inhibitors of kynurenine 3-hydroxylase. J. Med. Chem. 40 (26), 4378-4385 (1997).

12 Lee WS, Lee SM, Kim MK et al. The tryptophan metabolite 3-hydroxyanthranilic acid suppresses $T$ cell responses by inhibiting dendritic cell activation. Int. Immunopharmacol. 17(3), 721-726 (2013).

13 Fazio F, Lionetto L, Molinaro G et al. Cinnabarinic acid, an endogenous metabolite of the kynurenine pathway, activates type 4 metabotropic glutamate receptors. Mol. Pharmacol. 81(5), 643-656 (2012).

14 Sahm F, Oezen I, Opitz CA et al. The endogenous tryptophan metabolite and NAD + precursor quinolinic acid confers resistance of gliomas to oxidative stress. Cancer Res. 73(11), 3225-3234 (2013).

15 Vallerini GP, Amori L, Beato C et al. 2-aminonicotinic acid 1-oxides are chemically stable inhibitors of quinolinic acid synthesis in the mammalian brain: a step toward new antiexcitotoxic agents. J. Med. Chem. 56(23), 9482-9495 (2013). 